\title{
The Effects of Thermal Noise in a Rayleigh-Benard Cell Near Its First Convective Instability
}

\author{
H. van Beijeren ${ }^{1}$ and E. G. D. Cohen ${ }^{1,2}$
}

Received May 26, 1988

\begin{abstract}
Recent experiments by Meyer et al. on the onset of convection in a RayleighBenard cell near the first convective instability can be described as being caused by random noise terms in the currents of heat and momentum, but the amplitude of these terms turns out to be about $10^{5}$ times as large as would follow from standard fluctuating hydrodynamics.
\end{abstract}

KEY WORDS: Rayleigh-Benard cell; fluctuating hydrodynamics; convection, onset of; pattern formation.

\section{INTRODUCTION}

In recent experiments on the initial stages of pattern formation in a Rayleigh-Benard convection cell, Meyer et al. ${ }^{(1)}$ observed very irregular patterns. In these experiments, the fluid was subjected to a heat current that varied either linearly or periodically in time from below to above the onset of the first instability. If the side wall of the cylindrical cell was made of a material with a thermal diffusivity different from that of the enclosed fluid, a reproducible circular roll pattern was observed at the onset of convection. However, if the thermal diffusivities of side wall and fluid were the same, the pattern at onset was found to be quite irregular and irreproducible.

A plausible explanation would be that the onset of patterns is caused by stochastic fluctuations in the currents of heat and momentum. Adding the Landau-Lifshitz expressions for these fluctuations (ref. 2,

\footnotetext{
${ }^{1}$ Institute for Theoretical Physics, University of Utrecht, 3508 TA Utrecht, The Netherlands.
}

${ }^{2}$ Permanent address: The Rockefeller University, New York, New York 10021. 
Chapter XVII) to the hydroaynamic equations, Ahlers et al. ${ }^{(3)}$ calculated the resulting increase of the average convective current as a function of time. They employed slip boundary conditions at the wall of the vessel and assumed that the fluctuations only affect the single eigenmode of the hydrodynamic equations that determines the new stationary state above the instability. They found then that the noise level following from the Landau-Lifschitz fluctuations was about a factor $10^{-6}$ too small to explain their experimental observations.

The assumption of a single relevant mode is not realistic, however, since there are several modes that become unstable at Rayleigh numbers just above the critical Rayleigh number for onset of convection. Eventually, only one of these stabilizes, but at times near the onset time all of them may get excited. In this paper, we describe a calculation that takes into account the effects of all the modes that become unstable at or just above the critical Rayleigh number. In addition, we use the more realistic stick boundary conditions, appropriate for the experimental situation. Consequently, we find an enhancement of the effect of the fluctuations by a factor of about 40 compared to the previous estimate. This is still far too low for explaining the experimental results, so that one has to conclude that internal thermal noise cannot be the driving force for the onset of convection in the experiments by Meyer et al. A preliminary account of these results was given in ref. 4 .

\section{EIGENFUNCTIONS OF THE LINEARIZED FLUCTUATING HYDRODYNAMIC EQUATIONS}

Consider a fluid in a uniform gravitational field with gravitational acceleration $g$, between two horizontal plates located at $z_{1}=-d / 2$ and $z_{2}=d / 2$ and kept at temperatures $T_{1}>T_{2}$, respectively. For our calculations we will assume periodic boundary conditions in the $x$ and $y$ directions, although this does not agree with the experimental situation to be described. Later we will argue that the precise form of these lateral boundary conditions is unimportant.

For not too large temperature differences, the stationary solution of the hydrodynamic equations below the critical Rayleigh number $R_{c}$ consists of a vanishing velocity field and a temperature and a pressure field with uniform gradients in the $z$ direction. Besides being stationary, this solution is also stable. For Rayleigh numbers $R>R_{c}$ the same type of solution still is stationary, but it is no longer stable.

For small deviations from this stationary solution, both at subcritical and supercritical Rayleigh numbers, the hydrodynamic equations can be 
linearized around this solution. Collecting ${ }^{(5)}$ the deviations of the hydrodynamic fields from their stationary values into a vector

$$
\delta \mathbf{a}(\mathbf{r}, t)=\left(\begin{array}{c}
\delta p(\mathbf{r}, t) \\
\delta T(\mathbf{r}, t) \\
\mathbf{u}(\mathbf{r}, t)
\end{array}\right)
$$

one can write linearized fluctuating hydrodynamic equations as

$$
\frac{\partial}{\partial t} \delta \mathbf{a}(\mathbf{r}, t)=-\mathbf{h}(\mathbf{r}) \delta \mathbf{a}(\mathbf{r}, t)+\delta \mathbf{F}(\mathbf{r}, t)
$$

Here $\mathrm{h}$ is a linear differential operator, defined in ref. 5 , and $\delta \mathbf{F}$ is a stochastic noise term with vanishing stochastic average $\langle\delta \mathbf{F}(\mathbf{r}, t)\rangle$, where the average is taken over a Gaussian distribution of $\delta \mathbf{F}$. More explicitly, $\delta \mathbf{F}$ can be written in the form

$$
\delta \mathbf{F}(\mathbf{r}, t)=\left(\begin{array}{c}
-[(\gamma-1) / \alpha T] \mathbf{\nabla} \cdot \delta \mathbf{Q}(\mathbf{r}, t) \\
-\left(1 / \rho c_{v}\right) \nabla \cdot \delta \mathbf{Q}(\mathbf{r}, t) \\
(1 / \rho) \nabla \cdot \delta \mathrm{T}(\mathbf{r}, t)
\end{array}\right)
$$

Here $\rho$ is the mass density, $\gamma=c_{p} / c_{v}$ with $c_{p}$ and $c_{v}$ the specific heat per unit mass at constant pressure and volume, respectively, $\alpha$ is the thermal expansion coefficient, $\delta \mathbf{Q}$ is the random heat flux, and $\delta \mathrm{T}$ is the random stress tensor. The covariances of the random currents are given by the LandauLifshitz expressions ${ }^{(2,5)}$

$$
\begin{aligned}
\left\langle\delta Q_{i}\left(\mathbf{r}_{1}, t_{1}\right) \delta Q_{j}\left(\mathbf{r}_{2}, t_{2}\right)\right\rangle & =2 k_{\mathrm{B}} T^{2} \lambda \delta_{i j} \delta\left(\mathbf{r}_{1}-\mathbf{r}_{2}\right) \delta\left(t_{1}-t_{2}\right) \\
\left\langle\delta Q_{i}\left(\mathbf{r}_{1}, t_{1}\right) \delta T_{k l}\left(\mathbf{r}_{2}, t_{2}\right)\right\rangle= & 0 \\
\left\langle\delta T_{i j}\left(\mathbf{r}_{1}, t_{1}\right) \delta T_{k l}\left(\mathbf{r}_{2}, t_{2}\right)\right\rangle= & 2 k_{\mathrm{B}} T\left[\eta \left(\delta_{i k} \delta_{j l}+\delta_{i l} \delta_{j k}\right.\right. \\
& \left.\left.-\frac{2}{3} \delta_{i j} \delta_{k l}\right)+\zeta \delta_{i j} \delta_{k l}\right] \delta\left(\mathbf{r}_{1}-\mathbf{r}_{2}\right) \delta\left(t_{1}-t_{2}\right)
\end{aligned}
$$

with $\lambda, \eta$, and $\zeta$ the thermal conductivity, the shear viscosity, and the bulk viscosity, respectively, and $k_{\mathrm{B}}$ Boltzmann's constant. These hydrodynamic equations have to be supplemented by boundary conditions at the walls of the vessel. Most experimental situations correspond to stick boundary conditions, i.e., vanishing fluid velocity and no temperature jump at the walls, but in hydrodynamic calculations one often also uses slip boundary conditions, i.e., no tangential stresses and no temperature gradient perpendicular to the walls. The form of the hydrodynamic equations can be simplified by expansing $\delta \mathbf{a}$ in right eigenfunctions of the operator $h$. We 
will only be interested in those eigenfunctions that become unstable for $R$ equal to or just above $R_{c}$. These can be labeled as $\mathbf{a}_{\mathbf{k} / n}^{R}$, with $\mathbf{k}_{\| \mid}$their wave number in the horizontal plane and $n$ labeling their $z$ dependence. The hydrodynamic fields can be expressed in terms of the $\mathbf{a}_{\mathbf{k}_{\mid} \|^{n}}^{R}$ as

$$
\delta \mathbf{a}(\mathbf{r}, t)=\sum_{\mathbf{k}_{|| n}} a_{\mathbf{k}_{\mid n} n}(t) \mathbf{a}_{\mathbf{k}_{|| n}^{R}}^{R}(\mathbf{r})
$$

The coefficients $\alpha_{\mathbf{k}_{|| n}}(t)$ can be obtained as

$$
\alpha_{\mathbf{k}_{\| n} n}(t)=\left(\mathbf{a}_{\mathbf{k}_{\| n}}^{L}, \mathbf{a}\right)
$$

where the inner product $(\cdot, \cdot)$ is defined through $(f, g)=\int_{V} f^{*}(\mathbf{r}) g(\mathbf{r}) d \mathbf{r}$ and the $\mathbf{a}_{\mathbf{k} \mid n}^{L}$ are the left eigenfunctions of $h$, satisfying

$$
\left(\mathbf{a}_{\mathbf{k}_{\mid}{ }^{n}}^{L}, \mathbf{a}_{\mathbf{k}_{\mid}^{\prime} n^{\prime}}^{R}\right)=\delta_{\mathbf{k}_{||} \mid \mathbf{k}_{\|}^{\prime}} \delta_{n n^{\prime}}
$$

Since near $R=R_{c}$ only modes with $n=1$ are relevant, we restrict ourselves to these modes. Taking the inner product of Eq. (2) with $\mathbf{a}_{\mathbf{k}_{\| \mid 1}}^{L}$, one reduces the hydrodynamic equations to a set of linear stochastic differential equations for the coefficients $\alpha_{\mathbf{k}||}(t)$ of the form ${ }^{3}$

$$
\frac{\partial \alpha_{\mathbf{k}_{\|}}(t)}{\partial t}=-\hat{\lambda}_{\mathbf{k}_{\|}}(R(t)) \alpha_{\mathbf{k}_{\|}}(t)+f_{\mathbf{k}_{\|}}(t)
$$

where we suppressed the subscript 1 , as we will do in the rest of the main text.

Near $R_{c}$ the eigenvalue $\hat{\lambda}_{\mathbf{k}_{\|}}$depends on $R$ according to ${ }^{(5)}$

$$
\lambda_{\mathbf{k}_{\| \mid}}=-\frac{1}{\tau_{0} t_{D}}\left[\frac{R_{c}-R}{R_{c}}+A^{2}\left(\frac{k_{\| \mid}-k_{\| c}}{k_{\| c}}\right)^{2}\right]
$$

Here $t_{D}$ is the thermal diffusion time, $t_{D}=d^{2} / D_{T}$, with $D_{T}=\lambda / \rho c_{p}$ and $\rho$ is the mass density. $k_{\| c}$ is the value of $k_{||}$for the modes that become unstable at $R=R_{c}$ and $\tau_{0}$ is a dimensionless constant depending on the Prandtl number $P=v / D_{T}$, with $v=\eta / \rho$. In the Appendix, we give an expression for

${ }^{3}$ If the Rayleigh number depends on time, the modes $\mathbf{a}_{\mathbf{k}_{|| n}}^{L}$ and $\mathbf{a}_{\mathbf{k}_{\| n}^{R}}^{R}$ depend on time as well, since they are functions of the Rayleigh number. This does not change Eq. (8), however. For, in this case there is an additional contribution to $\partial \alpha_{i} / \partial t\left(i=\mathbf{k}_{\mid ! n}\right)$ of the form $(d R / d t)\left(\partial \mathbf{a}_{i}^{L} / \partial R\right.$, $\left.\sum_{j} \alpha_{j} \mathbf{a}_{j}^{R}\right)$. The term with $j=i$ vanishes, since $\partial \mathbf{a}_{i}^{L} / \partial R$ is orthogonal to $\mathbf{a}_{i}^{R}$ by virtue of conservation of normalization. Because a change in $R$ does not affect the $x$ and $y$ dependence of the $\mathbf{a}_{i}^{L}$, the modes $j$ that could contribute must have the same $\mathbf{k}_{\| \mid}$as $\mathbf{a}_{i}^{R}$, hence a different $n$, i.e., $n \neq 1$. But these modes do not contribute to (8) either, since they are not excited and hence the corresponding $\alpha_{j}$ vanish. 
$\tau_{0}$ in terms of the eigenfunctions of the hydrodynamic operator $h$. Finally, $\Lambda^{2}$ is another dimensionless quantity, which for stick boundary conditions is $\sim 1.46$. The fluctuating force $f_{\mathbf{k} \|}(t)$ in $(8)$ is again a Gaussian random noise term with vanishing average. Its variance is obtained by substituting (5) into the left-hand side of (2), taking the inner product of this equation with $\mathbf{a}_{\mathbf{k}_{\mid}}^{L}$, multiplying the resulting equation with its complex conjugate, and employing (4). The result is

$$
\left\langle f_{\mathbf{k}||}^{*}(t) f_{\mathbf{k}||}\left(t^{\prime}\right)\right\rangle=2 F \delta\left(t-t^{\prime}\right) \delta_{\mathbf{k} \mid \mathbf{k}_{\|}^{\prime}}
$$

with

$$
F=\frac{k_{\mathrm{B}} T D_{T}}{\rho d^{2} \tau_{0} V}\left(1+\frac{\alpha g T}{c_{p} d T / d z}\right)
$$

In the Appendix, the derivation of this result is considered in more detail.

\section{TIME EVOLUTION OF AMPLITUDES AND CONVECTIVE CURRENT}

By integrating (8) from an initial time $t_{0}$ up to $t$, multiplying the result by its complex comjugate, and employing (10a), one finds that the average squared amplitude $\left\langle\left|\alpha_{\mathbf{k}}(t)\right|^{2}\right\rangle$ satisfies an ordinary differential equation of the form

$$
\frac{\partial\left\langle\left|\alpha_{\mathbf{k}_{\|}}(t)\right|^{2}\right\rangle}{\partial t}=-2 \lambda_{\mathbf{k}_{\|}}(R)\left\langle\left|\alpha_{\mathbf{k}_{\|}}(t)\right|\right\rangle^{2}+2 F
$$

The solution of this equation can be given straightforwardly and reads

$$
\left\langle\left|\alpha_{\mathbf{k}_{\mid} \mid}(t)\right|^{2}\right\rangle=\left\langle\left|\alpha_{\mathbf{k}_{i}}(t)\right|^{2}\right\rangle_{\text {det }}+\left\langle\left|\alpha_{\mathbf{k}_{\|}||}(t)\right|^{2}\right\rangle_{\text {st }}
$$

where the deterministic contribution is given by

$$
\left\langle\left|\alpha_{\mathbf{k}_{\mid}}(t)\right|^{2}\right\rangle_{\operatorname{det}}=\exp \left\{\int_{t_{0}}^{t} d \tau\left[-2 \lambda_{\mathbf{k}_{\|}}(R(\tau)]\right\}\left\langle\left|\alpha_{\mathbf{k}_{\mid} \mid}\left(t_{0}\right)\right|^{2}\right\rangle\right.
$$

and the stochastic contribution by

$$
\left\langle\left|\alpha_{\mathbf{k}_{\mid l}}(t)\right|^{2}\right\rangle_{\mathrm{st}}=2 \int_{t_{0}}^{t} d t^{\prime}\left\{\exp \int_{t^{\prime}}^{t} d \tau\left[-2 \lambda_{\mathbf{k}_{||}}(R(\tau))\right]\right\} F
$$

Since we will consider experiments in which the convective heat current $J_{\text {conv }}$ in the Rayleigh-Benard cell is measured, we express $J_{\text {conv }}$ in terms of 
the amplitudes $\left|\alpha_{\mathbf{k}_{i}}\right|^{2}$. In the Appendix, we show that the amplitude of the convective heat current at constant Rayleigh number can be expressed as

$$
J_{\text {conv }}=\frac{1}{d} \int d \mathbf{r} \rho c_{p} u_{z}(\mathbf{r}) \delta T(\mathbf{r})
$$

Note that, although for given $\mathbf{r}$ the convective heat current density $\mathbf{J}_{\text {conv }}(\mathbf{r})$ in general is composed of three contributions - the "obvious" convective heat current $\rho c_{p} u_{z}(\mathbf{r}) \delta T(\mathbf{r})$, a local conductive contribution $-\rho c_{p} D_{T} \partial \delta T / \partial z$, and a viscous heating contribution - their sum after integration over $x$ and $y$ does not depend on $z$ and is given by (13). If the Rayleigh number is varied slowly, i.e., on a characteristic time scale that is large compared to $\tau_{0} t_{D}$, as is the case in the above-mentioned experiments, (13) is an excellent approximation. Inserting (5) into (13) and expressing the resulting integral over $u_{z} \delta T$ in terms of the constant $\tau_{0}$ introduced in (9), one obtains the relation

$$
J_{\text {conv }}=\frac{S D_{T} \rho c_{p}}{\alpha g d^{2} \tau_{0}} \sum_{\mathbf{k}_{\|}}\left\langle\left|\alpha_{\mathbf{k}_{\|}}(t)\right|^{2}\right\rangle
$$

with $S$ the area of the cell. We will normalize $J_{\text {conv }}$ by dividing it by the conductive current $J_{0}$ of a stationary system at the onset of convection, which is of the form

$$
J_{0}=\rho c_{p} D_{T}\left(\frac{d T}{d z}\right)_{c}
$$

where $(d T / d z)_{c}$ is the temperature gradient at the critical Rayleigh number. Hence, using the explicit form of the Rayleigh number $R=$ $\alpha g(d T / d z) d^{4} / v D_{T}$, one finds

$$
\frac{J_{\text {conv }}}{J_{0} S}=\frac{d^{2}}{D_{T} v R_{c} \tau_{0}} \sum_{\mathbf{k}_{\| \mid}}\left\langle\left|\alpha_{\mathbf{k}_{\|}}(t)\right|^{2}\right\rangle
$$

where the time dependence obviously is determined by Eq. (12).

\section{EXPERIMENTAL APPLICATIONS}

In ref. 1, Meyer et al. describe two experiments, which can both be discussed on the basis of the equations developed above.

\subsection{The Ramping Experiment}

In this experiment, the total heat current at the lower plate of the Rayleigh-Benard cell is increased linearly with time. In Appendix $\mathrm{C}$ of 
ref. 3 , it was shown that under these conditions also the temperature difference between the plates, and therefore the Rayleigh number, increases linearly with time until the convective current becomes noticeable [see Eq. (C6) of ref. 3]. In that case, the time dependence of the Rayleigh number can be written as

$$
R(t)=R_{c}\left(1+\beta t / t_{D}\right)
$$

with the time origin chosen as the moment at which the critical Rayleigh number is reached. The constant $\beta$ is called the ramping rate. For initial Rayleigh numbers that are well below $R_{c}$, the time dependence of $J_{\text {conv }} / J_{0}$ is found from (16), (12), (10b), and (9) to be

$$
\begin{aligned}
\frac{J_{\text {conv }} \approx}{J_{0}} \sum_{\mathbf{k}} & \frac{2 F t_{D} d^{2}}{\tau_{0} v D_{T} R_{c}}\left(\frac{\pi \tau_{0}}{\beta}\right)^{1 / 2} \exp \frac{\beta}{\tau_{0}}\left\{\frac{t}{t_{D}}-\frac{A^{2}}{\beta}\left(\frac{k_{\|}-k_{\| c}}{k_{\| c}}\right)^{2}\right\}^{2} \\
& \times \Theta\left\{\frac{t}{t_{D}}-\frac{A^{2}}{\beta}\left(\frac{k_{\|}-k_{\| c}}{k_{\| c}}\right)^{2}\right\} \\
\approx & \sum_{\mathbf{k}} \frac{2 k_{\mathbf{B}} T t_{D}}{\rho V v R_{c}}\left(\frac{\pi}{\beta \tau_{0}^{3}}\right)^{1 / 2} \exp \frac{\beta}{\tau_{0}}\left\{\frac{t}{t_{D}}-\frac{A^{2}}{\beta}\left(\frac{k_{\|}-k_{\mid c}}{k_{\| c}}\right)^{2}\right\}^{2} \\
& \times \Theta\left\{\frac{t}{t_{D}}-\frac{A^{2}}{\beta}\left(\frac{k_{\|}-k_{\| c}}{k_{\| c}}\right)^{2}\right\}
\end{aligned}
$$

Here $\Theta(x)$, the unit step function, approximates the error function that results from (9) and (12b). The integral over $t^{\prime}$ in (12b) was approximated by a Gaussian integral from $-\infty$ to $\infty$, the second term between square brackets of $(10 \mathrm{~b})$ was neglected, since it is very small compared to unity under the experimental conditions considered. For large aspect ratios the summation over $\mathbf{k}_{\|}$may be approximated by an integral. ${ }^{4}$ The density of eigenfunctions in $\mathbf{k}_{\mid}$space equals $S / 4 \pi^{2}$. If one neglects the fourth-order term in $k_{\|}-k_{\| c}$ in the exponential, which is allowed for $t / t_{D} \gg 1$, another approximately Gaussian integral remains and one obtains for the ramping experiment

$$
\frac{J_{\mathrm{conv}}}{J_{0}} \approx\left(\frac{t_{D}}{2 \beta t}\right)^{1 / 2} \frac{t_{D} k_{\mathrm{B}} T a_{c}^{2}}{\Lambda \rho \tau_{0} d^{3} v R_{c}} \exp \left[\frac{\beta}{\tau_{0}}\left(\frac{t}{t_{D}}\right)^{2}\right]
$$

where $a_{c}=k_{\| c} d$.

\footnotetext{
${ }^{4}$ In this approximation the precise nature of the boundary conditions at the side walls indeed becomes unimportant.
} 


\subsection{The Oscillating Experiment}

In this case, $R(t)$ is a periodic function of time, which assumes alternatively values larger and smaller than $R_{c}$. A typical example is shown in Fig. 1. At the initial time $t_{0}$ the system is prepared in a particular mode, characterized, for example, by $\mathbf{k}_{\| \mid}$. Experimentally it is then found that whether or not the observed convection pattern is periodic in time depends quite sensitively on the time dependence of $R(t)$. In a series of experiments $^{(1)}\left(R-R_{c}\right) / R_{c}$ was modulated sinusoidally with amplitude $\delta$ around an average value $\varepsilon$. When $\delta$ and $\varepsilon$ were varied, the experiments could be understood by assuming that the initially imposed pattern persisted if $\left(R-R_{c}\right) / R_{c}$ never dropped below a certain threshold value, whereas it was replaced by a different, stochastically varying pattern if this quantity did drop below the threshold value.

Our conjecture is that the persistence of the initial pattern is determined by the minimum of the ratio of the deterministic amplitude of the initially imposed mode to the average amplitude of the modes generated stochastically by the noise in the system. These two quantities follow from (12a) and (12b), respectively, as

$$
\left\langle\left|\alpha_{\mathbf{k}_{\|}}(t)\right|^{2}\right\rangle_{\operatorname{det}}=\left\langle\left|\alpha_{\mathbf{k}_{\|}}\left(t_{1}\right)\right|^{2}\right\rangle \exp \left[-2 \int_{t_{1}}^{t} d \tau \lambda_{\mathbf{k}_{\|} \mid}(\tau)\right]
$$

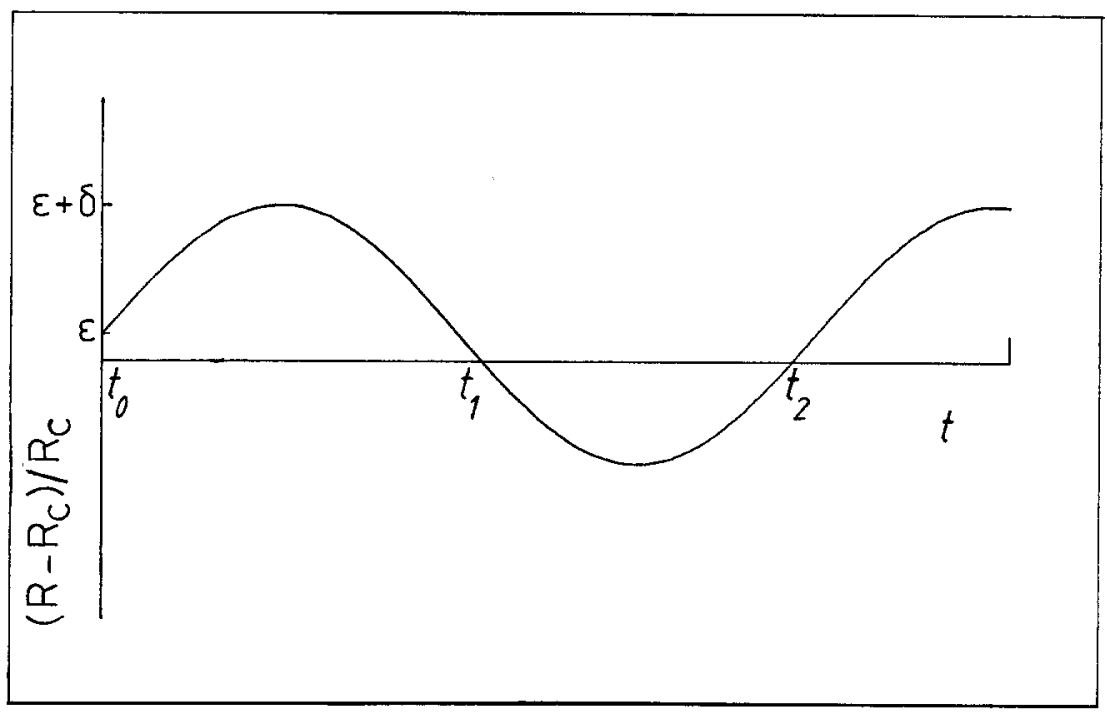

Fig. 1. Typical behavior of the Rayleigh number in time, in the ocillating experiment. 
and

$$
\left\langle\sum_{\mathbf{k}_{\|}^{\prime}}\left|\alpha_{\mathbf{k}_{i}^{\prime} \mid}(t)\right|^{2}\right\rangle_{\mathrm{st}}=\sum_{\mathbf{k}_{\mid}^{\prime}} \int_{t_{1}}^{t} d \tau \exp \left[-2 \int_{\tau}^{t} \lambda_{\mathbf{k}_{\mid}^{\prime}}\left(t^{\prime}\right) d t^{\prime}\right] F
$$

where $\left\langle\left|\alpha_{\mathbf{k}_{\|}}\left(t_{1}\right)\right|^{2}\right\rangle$ has to be determined from the hydrodynamic equations for $R>R_{c}$.

Comparison between our predictions and the experimental results of Meyer $e t$ al. is made in the next section.

\section{DISCUSSION}

\subsection{The Ramping Experiment}

In order to make a comparison with Meyer et al., we interpret, as they do, the expression (19) for the time dependence of the convective heat current in the ramping experiment as resulting from the excitation of an effective single mode. Then, the convective heat current is described by an equation similar to $(8)$, i.e.,

$$
\frac{\partial \alpha(t)}{\partial t}=\frac{1}{\tau_{0} t_{D}} \frac{R(t)-R_{c}}{R_{c}} \alpha(t)+f(t)
$$

with $\left\langle\alpha^{2}(t)\right\rangle=J_{\text {conv }} / J_{0},\langle f(t)\rangle=0$, and $\left\langle f(t) f\left(t^{\prime}\right)\right\rangle=2 F_{\text {eff }} \delta\left(t-t^{\prime}\right)$. To obtain agreement with (19), one has to choose a different value of $F_{\text {eff }}$ for each time $t_{0}$ and then so solve $(21)$ as though $F_{\text {eff }}$ had this value for all times; the result for $\left\langle\alpha^{2}(t)\right\rangle$ then has the correct value for $t=t_{0}$. The explicit form of $F_{\mathrm{eff}}$ is then

$$
F_{\mathrm{eff}}\left(t_{0}\right)=\frac{k_{\mathrm{B}} T a_{c}^{2}}{2 \Lambda \rho \tau_{0} d^{3} v R_{c}}\left(\frac{t_{D}}{2 \pi t_{0} \tau_{0}}\right)^{1 / 2}
$$

The time dependence $\sim t_{0}^{-1 / 2}$ is caused by the fact that the off-critical modes grow slightly more slowly (or decay a bit faster) than the critical ones. In ref. 3 , the time evolution of the convective curent is described by an equation of the form (21), but with an $f(t)$ that contains, instead of $F_{\text {eff }}{ }^{5}$

$$
F_{\mathrm{ACHS}}=\frac{k_{\mathrm{B}} T d^{2}}{D_{T}^{2} \rho d \tau_{0}(1+P) S t_{D}}\left(\frac{3}{R_{c}}\right)^{1 / 2}
$$

${ }^{5}$ This result follows from (4.20), (D20), and (D18) of ref. 3. An extra factor $t_{D}^{-1}$ in (23) results because $t$ in ref. 3 corresponds to $t / t_{D}$ in our notation. As a result, $f$ in ref. 3 corresponds to $f t_{D}$ here and $\delta\left(t-t^{\prime}\right)$ in ref. 3 to $t_{D} \delta\left(t-t^{\prime}\right)$ in our units. Hence, their $\vec{F}$ corresponds to $\tau_{0} t_{D} F_{\text {ACHS }}$. 
The main qualitative difference between (23) and (22) is that $F_{\mathrm{ACHS}}$ is inversely proportional to the horizontal area $S$ of the system, whereas for large systems our $F$ is independent of this quantity.

To compare (22) with the experiment on water in a cylindrical container described in ref. 1 , we used the parameters $T_{2}=2.99 \mathrm{~K}, T_{1}-T_{2}=$ $2.65 \mathrm{~K}, D_{T} \simeq 1.47 \times 10^{-3} \mathrm{~cm}^{2} / \mathrm{sec}, P=6.0, d=0.318 \mathrm{~cm} ; 3.18 \mathrm{~cm}$ for the radius of the cylinder, and the values $R_{c}=1707.8, a_{c}=3.117, A^{2}=1.46$, and $\tau_{0}^{-1}=19.65 P /(P+0.5117)=18$, valid for stick boundary conditions. Inserting these values into (22) for $t / t_{D}=1$, one finds that $\tau_{0} t_{D} F_{\text {eff }} \approx$ $4 \times 10^{-11}$, whereas $\tau_{0} t_{D} F_{\mathrm{ACHS}} \approx 10^{-12}$, i.e., about a factor 40 less. Experimentally, Meyer et al. obtained a value of about $6 \times 10^{-7}$ for this quantity, so that we have to conclude that the noise driving the pattern formation in this experiment is not internal thermal noise as described by the Landau-Lifschitz fluctuations.

It is not clear what the source of the noise driving the system could be. Mareschal and Kestemont ${ }^{(6)}$ describe computer simulations on a Rayleigh-Benard cell filled with hard disks in two dimensions, and suggest that the onset of pattern formation may be governed by thermal fluctuations at the walls of the system. However, in their system the ratio of the plate distance to the disk diameter is in the range between 10 and 100 , whereas it is on the order of $10^{7}$ in the real fluid experiments described above. Therefore, these boundary effects can be estimated to be about five orders of magnitude smaller than in the simulations of Mareschal and Kestemont, which makes them completely insignificant for the experiments of Meyer et al.

\subsection{The Oscillating Experiment}

Consider a sinusoidal modulation of the Rayleigh number of the form

$$
\left[R(t)-R_{c}\right] / R_{c}=\varepsilon+\delta \sin \Omega t / t_{D}
$$

as pictured in Fig. 1. In order to predict the type of behavior seen in the experiments, we want to determine the strengths of the deterministic and the stochastic currents, using Eqs. (11), (12), and (14). We first calculate $J_{\text {conv }}^{\text {det }}$ for $t_{1}$ (Fig. 1).

For large enough amplitudes and not too large frequency $\Omega$ the system will be close to a convective stationary state during the major part of the time intervals where $R>R_{c}$. Then the fluctuating terms in the hydrodynamic equations can be ignored and the convective current follows a deterministic equation of the form

$$
\frac{\partial}{\partial t} J_{\text {conv }}^{\mathrm{det}}(t)=\frac{-2}{\tau_{0} t_{D}}\left[J_{\text {conv }}^{\mathrm{det}}(t)-J_{\text {conv }}^{0}(R(t))\right]
$$


where $J_{\text {conv }}^{0}(R)$ is the stationary convective current at Rayleigh number $R>R_{c}$. Here we assumed that the slowest decay rate of perturbations around the stable stationary state is given by $\left(1 / \tau_{0} t_{D}\right)\left(R-R_{c}\right)$, as is the case for slip boundary conditions. ${ }^{(8)}$ From Eq. (2.30) of ref. 4 , we take as an approximation for $J_{\text {conv }}^{0}(R)$

$$
J_{\text {conv }}^{0}(R) / J_{0}=0.84\left(R-R_{c}\right)
$$

Provided $2 \Omega / \tau_{0} \gg 1$, the current at time $t_{1}$ is determined mainly by the values of $R(t)$ during a small time interval preceding $t_{1}$. In this case, a linear approximation

$$
R(t) \approx\left(t_{1}-t\right) \beta(\varepsilon, \delta)
$$

is allowed for $t<t_{1}$, with

$$
\beta(\varepsilon, \delta)=-t_{D}\left(\frac{d R(t)}{d t}\right)_{t=t_{1}}=\delta\left[1-\left(\frac{\varepsilon}{\delta}\right)^{2}\right]^{1 / 2} \Omega
$$

An approximate solution of (25) for $t=t_{1}$ is then

$$
J_{\text {conv }}^{\text {det }}\left(t_{1}\right) / J_{0} \approx 0.42\left[\pi \tau_{0} \beta(\varepsilon, \delta)\right]^{1 / 2}
$$

Using (28) as the starting value for (12a), we find for $t>t_{2}$

$$
\begin{aligned}
\frac{J_{\text {conv }}^{\text {det }}(t)}{J_{0}} \approx & 0.42\left[\pi \tau_{0} \beta(\varepsilon, \delta)\right]^{1 / 2} \exp \frac{-4 \delta}{\tau_{0} \Omega}\left\{\left[1-\left(\frac{\varepsilon}{\delta}\right)^{2}\right]^{1 / 2}-\frac{\varepsilon}{\delta} \arccos \frac{\varepsilon}{\delta}\right\} \\
& \times \exp \frac{2}{\tau_{0} t_{D}} \int_{t_{1}}^{t} d \tau \frac{R(\tau)-R_{c}}{R_{c}}
\end{aligned}
$$

Next we calculate $J_{\text {conv }}^{\text {st }}$ for $t>t_{2}$. A linear approximation, similar to (27), for the time behavior of $R$ in the neighborhood of $t_{2}$ yields a stochastic convective current $J_{\text {conv }}^{\text {st }}$ of average strength

$$
\begin{aligned}
J_{\text {conv }}^{\mathrm{st}}(t) / J_{0} \approx & 2 F_{\text {eff }} t_{D}\left[\pi \tau_{0} / \beta(\varepsilon, \delta)\right]^{1 / 2} \\
& \times \exp \left(2 / \tau_{0} t_{D}\right) \int_{t_{1}}^{t} d \tau\left[R(\tau)-R_{c}\right] / R_{c}
\end{aligned}
$$

From (29) and (30) we conclude that $J^{\text {det }}$ and $J^{\text {st }}$ are of equal strengths if $\delta$ and $\varepsilon$ are related by the equation

$$
\delta=\frac{t_{0} \Omega}{4\left\{\left[1-(\varepsilon / \delta)^{2}\right]^{1 / 2}-(\varepsilon / \delta) \arccos (\varepsilon / \delta)\right\}} \log \frac{0.21 \beta(\varepsilon, \delta)}{F_{\text {eff }} t_{D}}
$$


In Fig. 2, we plot relation (31) for $\Omega=1$, with $\tau_{0}^{-1}=18$ and the value $\tau_{0} t_{D} F_{\text {eff }}=6 \times 10^{-7}$, as obtained by Meyer $e t$ al. in the ramping experiment. In addition, we draw in the experimental points indicating the boundary between deterministic and stochastic behavior, as given in Fig. 5 of ref. 1. Obviously these points are quite close to the curve defined by (31); hence the results of the ramping experiment and those of the oscillating experiment are consistent with each other, as has been remarked by Swift and Hohenberg ${ }^{(9)}$ on the basis of a different calculation.

We based all our considerations on the linearized equations (2). One may wonder how the nonlinearities that were left out of our analysis (cf. ref. 3 ) would affect the results. We first note that the magnitude of the nonlinear contribution that should be added to (8) equals that of the linear term only when the system is in its convecting stationary state. The ratio of the nonlinear contribution to the linear one can be estimated by the ratio of the actual convective current to its stationary value. This ratio remains small during the onset period. Second, the effect of the nonlinear term usually is to suppress the amplitude, as can be seen, e.g., from Eqs. (2.1)-(2.2) of ref. 3. Hence, inclusion of this term will not improve our estimates.

Even though we had to conclude that the experiments of Meyer et al. cannot be explained as being driven by internal thermal noise, the theory

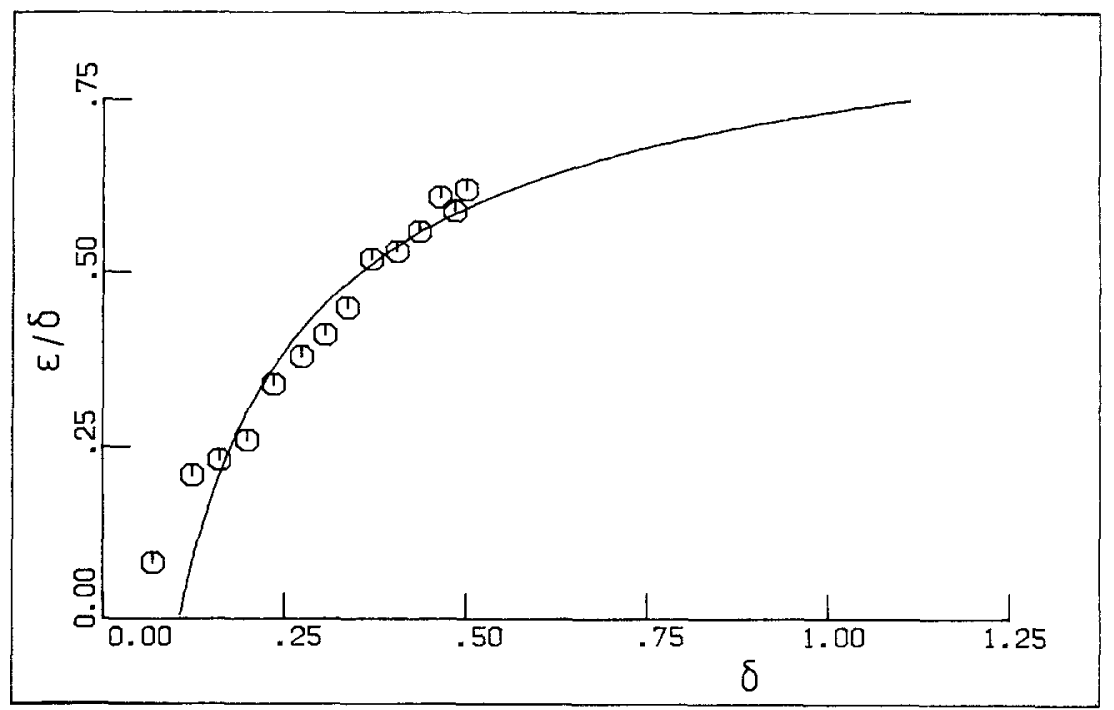

Fig. 2. The line gives the locus of points in the $\varepsilon / \delta$ versus $\delta$ plane for which the deterministic and the stochastic convective currents reach equal amplitudes. The data points are experimental points, taken from Fig. 5 of ref. 1, that mark the boundary between periodic and stochastic behavior. 
developed here may be relevant to the experiment. For, as long as the stochastic contributions to the heat and momentum current can be described as Gaussian white noise with a variance $F$ that is approximately constant for all modes that become unstable for $R$ just above $R_{c}$, all our equations can be applied, except for $(10 \mathrm{~b})$, which must be replaced by the appropriate value for $F$.

We remark that experiments similar to those carried out here for a Rayleigh-Benard cell could also be conceived for Taylor-Couette flow. If then, as in the Rayleigh-Benard case, there are many different critical modes, the possibility of stochastic irreproducible patterns like those observed by Meyer $e t$ al. exists.

\section{APPENDIX}

In ref. 7, the hydrodynamic operator $h(r)$ is derived as ${ }^{6}$

$$
h(\mathbf{r})=\left[\begin{array}{ccc}
0 & -\frac{\alpha \gamma}{\chi_{T}} D_{T} \Delta & \frac{\gamma}{\chi_{T}} \nabla-g \rho \mathbf{e}_{z} \\
0 & -\gamma D_{T} \Delta & \frac{\gamma-1}{\alpha} \nabla+\frac{d T}{d z} \hat{e}_{z} \\
\frac{1}{\rho} \nabla+g \chi_{T} \mathbf{e}_{z} & -g \alpha \mathbf{e}_{z} & -v \Delta 1-\left(\Gamma_{i}-v\right) \nabla \nabla
\end{array}\right]
$$

Here $\Delta$ is the Laplacian, $\chi_{T}$ the isothermal compressibility, and $\Gamma_{l}$ the longitudinal viscosity. The fluctuating hydrodynamic fields in a RayleighBenard cell relevant in this paper are superpositions of so-called viscoheat modes, which are a specific type of right eigenfunction of the operator $h(r)$ satisfying the boundary conditions. They generally are of the form [see Eq. (7.5) of ref. 7]

$$
\mathbf{a}_{k_{|| n} n}^{R}(\mathbf{r})=\left(\begin{array}{c}
0 \\
T_{k_{\| n}^{R}(z)}^{R}(z) V_{k_{\mid ! n}^{R}}^{R}(z)
\end{array}\right) \exp \left(i \mathbf{k}_{\| \mid} \cdot \mathbf{r}_{\| \mid}\right)
$$

where $n$ is a discrete index labeling viscoheat modes with different $z$ dependence but the same $\mathbf{k}_{||}$. The functions $T_{k_{||} n}^{R}(z)$ and $V_{k_{\mid n}}^{R}(z)$ are of the form

$$
\begin{aligned}
& T_{k_{\mid} \mid n}^{R}(z)=\frac{v}{\alpha g d^{4} k_{||}^{2}} \Theta_{n}\left(\frac{z}{d}\right) \\
& V_{k_{||} n}^{R}(z)=W_{n}\left(\frac{z}{d}\right)
\end{aligned}
$$

${ }^{6}$ Actually, Eq. (5.13) of ref. 7 gives the Fourier transform of this operator with respect to $x$ and $y$. 
respectively [see Eqs. (4.10) of ref. 5]. For $n=1$, which describes the only modes of importance for $R$ near $R_{c}$, the specific forms of these functions for stick and slip boundary conditions, respectively, are given in Eqs. (6.21)-(6.22) of ref. 5. The corresponding left eigenvectors of $h(r)$ are given in Eq. (7.5) of ref. 7 as

$$
\mathbf{a}_{\mathbf{k}_{|| n} n}^{L}(\mathbf{r})=\left(\begin{array}{c}
-\left(\alpha T / \rho c_{p}\right) T_{k_{|| n}}^{L}(z) \\
T_{k_{\| n}}^{L}(z) \\
\left(1 / k_{\| \mid}^{2}\right) \nabla \times\left(\nabla \times e_{z}\right) V_{k_{|| n}}^{L}(z)
\end{array}\right) \exp \left(i \mathbf{k}_{\|} \cdot \mathbf{r}_{\|}\right)
$$

with $T_{k_{\| n} n}^{L}$ and $V_{k_{\| n} n}^{L}$ specified in (4.10) of ref. $5 \mathrm{as}^{7}$

$$
\begin{aligned}
& T_{k_{||} n}^{L}(z)=-\frac{1}{V} \frac{v}{(d T / d z) d^{4} k^{2}} \Theta_{n}^{*}\left(\frac{z}{d}\right) \\
& V_{k_{||} n}^{L}(z)=\frac{1}{V} W_{n}^{*}\left(\frac{z}{d}\right)
\end{aligned}
$$

where the star denotes complex conjugation. The eigenvalue of the critical viscoheat mode at $R=R_{c}$ equals zero. In first-order perturbation theory the deviation of $\mathrm{h}(\mathbf{r})$ from its form at $R=R_{c}$ is given by

$$
\delta \mathrm{h}(\mathbf{r})=\left(\begin{array}{ccc}
0 & 0 & 0 \\
0 & 0 & {\left[\left(R-R_{c}\right) / R_{c}\right](d T / d z)_{c} \mathbf{e}_{z}} \\
0 & 0 & 0
\end{array}\right)
$$

Hence the perturbation of the eigenvalue of the critical viscoheat mode is

$$
\begin{aligned}
-\lambda_{k_{\| \mid}}(R) & =\left(\mathbf{a}_{\mathbf{k}_{\| \mid c}^{L}}^{L} \delta \mathrm{h}, \mathbf{a}_{\mathbf{k}_{||}^{R}}^{R}\right) \\
& =\frac{v\left(R-R_{c}\right)}{d^{2} a_{c}^{2} R_{c}} \int_{-1 / 2}^{1 / 2} \Theta(x) W(x) d x
\end{aligned}
$$

where we used (A.2)-(A.5) and the property

$$
\mathbf{e}_{z} \cdot\left(\nabla \times\left(\nabla \times \mathbf{e}_{z}\right)\right)\left[\exp \left(i \mathbf{k}_{\|} \cdot \mathbf{r}_{\|}\right)\right] f(z)=-k_{\|}^{2} f(z)
$$

Comparison to (9) yields

$$
\frac{1}{\tau_{0}}=\frac{P}{a_{c}^{2}} \int_{-1 / 2}^{1 / 2} \Theta(x) W(x) d x
$$

\footnotetext{
${ }^{7}$ The factor $(2 \pi)^{2} d$ for the infinite system treated in ref. 5 has been replaced here by $V$.
} 
The stochastic force $f_{\mathbf{k}}(t)$ introduced in (8) follows from (2) by taking the inner product with $\mathbf{a}_{\mathbf{k}_{\mid 1}}^{L}$ as

$$
f_{\mathbf{k}_{\|}}(t)=\left(\mathbf{a}_{\mathbf{k}_{\|} \mid}^{L}, \delta \mathbf{F}(\mathbf{r}, t)\right)
$$

Inserting (3) for $\delta \mathbf{F}$ and (A.4), (A.5) for $\mathbf{a}_{\mathbf{k}_{\| 1}}^{L}$, one obtains an expression for the variance $\left\langle f_{\mathbf{k}_{\|}}^{*}(t) f_{\mathbf{k}||}\left(t^{\prime}\right)\right\rangle$ that can be evaluated through somewhat lengthy but straightforward calculations. The result is given in Eq. (10).

Finally, we want to derive Eq. (13) for the convective heat current. Our starting point is the following expression for the local convective current density (ref. 2, Chapter V):

with

$$
\mathbf{J}_{q}=\mathbf{J}_{\text {conv }}+\mathbf{J}_{\text {cond }}+\mathbf{J}_{\mathrm{vh}}
$$

$$
\begin{aligned}
\mathbf{J}_{\text {conv }} & =\rho c_{p} \mathbf{u} \delta T \\
\mathbf{J}_{\text {cond }} & =-\rho c_{p} D_{T} \nabla \delta T \\
\mathbf{J}_{\mathbf{v h}} & =\eta\left(\nabla \frac{1}{2} u^{2}+\mathbf{u} \cdot \nabla \mathbf{u}\right)
\end{aligned}
$$

where we used that $\nabla \cdot \mathbf{u}=0$ for viscoheat modes. Inserting (A.10) into the equation $\rho c_{p} \partial \delta T / \partial t=-\operatorname{div} \mathbf{J}_{q}$ and assuming $\rho, c_{p}, D_{T}$, and $\eta$ to be constant, one obtains the following result for the time evolution of $\delta T$ :

$$
\frac{\partial \delta T}{\partial t}=D_{T} \Delta \delta T-\nabla \cdot\left[\mathbf{u}\left(T_{0}+\delta T\right)+\frac{\eta}{\rho c_{p}}\left(\nabla \frac{1}{2} u^{2}+\mathbf{u} \cdot \nabla \mathbf{u}\right)\right]
$$

where $T_{0}$ is the conductive temperature field that results from a uniform gradient in the $z$ direction. The boundary conditions are $\mathbf{u}=0$ and $\delta T=0$ at the boundaries. Averaging Eq. (A.11) over $x$ and $y$, since we are only interested in the average of $\mathbf{J}_{q}$ over $x$ and $y$, denoting such averages by a bar, and introducing the scaled variable $\zeta=z / d$, we can make a Fourier representation of $\overline{\delta T}$ as

$$
\overline{\delta T(\zeta, t)}=\sum_{n=1}^{\infty}\left[\gamma_{n}(t) \sin 2 \pi n \zeta+\delta_{n}(t) \cos (2 n-1) \pi \zeta\right]
$$

where we took account of the boundary condition $\overline{\delta T}=0$ for $\zeta= \pm 1 / 2$. Applying a similar Fourier transform to the right-hand side of (A.11), one obtains the set of equations

$$
\begin{array}{r}
{\left[\frac{\partial}{\partial t}+\frac{(2 n \pi)^{2}}{t_{D}}\right] \gamma_{n}(t)=C_{n}(t)} \\
\left\{\frac{\partial}{\partial t}+\frac{[(2 n-1) \pi]^{2}}{t_{D}}\right\} \delta_{n}(t)=D_{n}(t)
\end{array}
$$


with

$$
\begin{aligned}
C_{n}(t)= & -2 \int_{-1 / 2}^{1 / 2} d \zeta \sin 2 \pi n \zeta \frac{\partial}{\partial z} \overline{u_{z} \delta T(z, t)} \\
= & \frac{4 \pi n}{d} \int_{-1 / 2}^{1 / 2} d \zeta \cos 2 \pi n \zeta \overline{u_{z} \delta T(z, t)} \\
D_{n}(t)= & -\frac{2 \eta}{\rho c_{p}} \int_{-1 / 2}^{1 / 2} d \zeta \cos (2 n-1) \pi \zeta \overline{\nabla \cdot\left\{\nabla \frac{1}{2} u^{2}+\mathbf{u} \cdot \nabla \mathbf{u}\right\}} \\
= & \frac{-2(2 n-1) \pi \eta}{\rho c_{p} d} \int_{-1 / 2}^{1 / 2} d \zeta \sin (2 n-1) \pi \zeta \\
& \times\left\{\frac{\left.\frac{\partial\left(\frac{1}{2} u^{2}(z, t)\right)}{\partial \zeta}+u_{z} \frac{\partial u_{z}(z, t)}{\partial \zeta}\right\}}{\partial \zeta}\right.
\end{aligned}
$$

The solutions of (A.13) and (A.14) read

$$
\begin{aligned}
\gamma_{n}(t)= & \left\{\exp \left[\frac{-(2 n \pi)^{2}}{t_{D}}\left(t-t_{0}\right)\right]\right\} \gamma_{n}\left(t_{0}\right) \\
& +\int_{t_{0}}^{t} d \tau\left\{\exp \left[\frac{-(2 n \pi)^{2}}{t_{D}}(t-\tau)\right]\right\} C_{n}(\tau) \\
\delta_{n}(t)= & \left\{\exp \left[\frac{-[(2 n-1) \pi]^{2}}{t_{D}}\left(t-t_{0}\right)\right]\right\} \delta_{n}\left(t_{0}\right) \\
& +\int_{t_{0}}^{t} d \tau\left\{\exp \left[\frac{-[(2 n-1) \pi]^{2}}{t_{D}}(t-\tau)\right]\right\} D_{n}(\tau)
\end{aligned}
$$

where $\gamma_{n}\left(t_{0}\right)$ and $\delta_{n}\left(t_{0}\right)$ are the initial values of $\gamma_{n}(t)$ and $\delta_{n}(t)$, respectively.

For a stationary system, $C_{n}(\tau)$ and $D_{n}(\tau)$ are constant, so that then $t_{0}$ may be replaced by $-\infty$ and the integrals over $\tau$ can be performed immediately.

For a quasistationary system, i.e., a system in which the typical time scale for variations in the hydrodynamic fields is $>t_{D} / \pi^{2}$, we may replace $C_{n}(\tau)$ and $D_{n}(\tau)$ in (A.17) by $C_{n}(t)$ and $D_{n}(t)$, respectively, obtaining the approximate results

$$
\begin{aligned}
& \gamma_{n}(t) \approx \frac{t_{D}}{(2 n \pi)^{2}} C_{n}(t) \\
& \delta_{n}(t) \approx \frac{t_{D}}{[(2 n-1) \pi]^{2}} D_{n}(t)
\end{aligned}
$$


Inserting (A.18) into (A.12) and that equation in turn into (A.10) leads to the following expression for $\mathbf{J}_{\text {cond }}$ :

$$
\begin{aligned}
\overline{\mathbf{J}_{\text {cond }}(z, t)}= & -\mathbf{e}_{z} \rho c_{p} d^{2} \sum_{n=1}^{\infty}\left[\frac{1}{2 n \pi d} \cos 2 \pi n \zeta C_{n}(t)\right. \\
& \left.-\frac{1}{(2 n-1) \pi d} \sin (2 n-1) \pi \zeta D_{n}(t)\right]
\end{aligned}
$$

where we used the definition $t_{D}=d^{2} / D_{T}$.

Furthermore, it follows from (A.10) and (A.15b) and that $\mathbf{u} \delta T$ is an even function of $z$, that the average of the purely convective current is given by

$$
\overline{\mathbf{J}_{\text {conv }}(z, t)}=\rho c_{p} \mathbf{e}_{z} \sum_{n=0}^{\infty} \frac{d}{2 \pi n} C_{n}(t)
$$

while (A.10) and (A.16b) give that the viscous heating contribution is

$$
\overline{\mathbf{J}_{\mathrm{vb}}(z, t)}=\rho c_{p} \mathbf{e}_{z} \sum_{n=0}^{\infty} \frac{d}{(2 n-1) \pi} D_{n}(t)
$$

Adding up the contributions (A.19)-(A.21), one arrives at the result of Eq. (13). For simplicity, the bars on $\mathbf{J}_{\text {conv }}$ have been omitted in the text.

\section{ACKNOWLEDGMENTS}

The authors are most indebted to G. Ahlers, D. S. Cannell, and C. W. Meyer for drawing their attention to this problem and for many stimulating discussions. They also gratefully acknowledge helpful discussions with Dr. R. Schmitz and Prof. Th. W. Ruygrok and they thank J. A. Leegwater for his help in preparing the figures and Diane M. Lott for typing the manuscript. This research was supported in part by the National Science Foundation under grant PHY82-17853, supplemented by funds from the National Aeronautics and Space Administration at the University of California at Santa Barbara. One of the authors (E.G.D.C.) acknowledges also support under Department of Energy contract DE-AC02-81ER10807. 


\section{REFERENCES}

1. C. W. Meyer, G. Ahlers, and D. S. Cannell, Phys. Rev. Lett. 59:1577 (1987).

2. L. D. Landau and E. M. Lifshitz, Fluid Mechanics (Pergamon, New York, 1959).

3. G. Ahlers, M. C. Cross, P. C. Hohenberg, and S. Safran, J. Fluid Mech. 110:297 (1981).

4. H. van Beijeren and E. G. D. Cohen, Phys. Rev. Lett. 60:1208 (1988).

5. R. Schmitz and E. G. D. Cohen, J. Stat. Phys. 40:431 (1985).

6. M. Mareschal and E. Kestemont, J. Stat. Phys. 48:1187 (1987).

7. R. Schmitz and E. G. D. Cohen, J. Stat. Phys. 39:285 (1985).

8. T. R. Kirkpatrick and E. G. D. Cohen, J. Stat. Phys. 33:639 (1983).

9. J. B. Swift and P. C. Hohenberg, Phys. Rev. Lett. 60:75 (1988). 